\section{PORTION OF A LECTURE}

\author{
DIAGNOSIS BETWEEN GENERAL PARESIS AND \\ PROGRESSIVE LOCOMOTOR ATAXY. \\ Delivered at University College, Summer Session, 1873.
}

By W. H. O. S A N K E Y, M.D.Lond., F.R.C.P., Lecturer on Mental Diseases at the College.

I HAVE formerly, when speaking of the diagnosis of general paresis (or general paralysis of the insane), satisfied myself with but a slight allusion to locomotor ataxy; believing that the phenomena were sufficiently distinct in character in each of these diseases to prevent any confusion between them, and such, indeed, is the case in a large majority of the examples met with.

The word ataxy literally means want of order, and, when applied to movements, it is called locomotor ataxy; a condition of such ataxy is met with in the insane occasionally as a symptom, and occurs as an accidental complication, just as we have also various other kinds of derangement of the nerve-function, as anæsthesia, convulsion, partial and local paralysis, etc. I think that in some of such cases the ataxy has been engrafted upon the mental disease ; in others, perhaps the insanity has occurred in an ataxic individual. I had a patient whose ataxic symptoms were strongly marked, and could, I think, be traced to a cause occurring subsequently to the attack of insanity. The patient was taken with the usual symptoms of melancholia with suicidal propensities, and she succeeded in throwing herself out of her first-floor window, and fell vertically upon her sacrum. Ataxic symptoms gradually appeared and increased, and she died about thirteen years after the commencement of the insanity. In a second case, the history, as gleaned several years after the events occurred, seemed to show a similar origin and course. But it is not so much perhaps these cases, with ataxy occurring symptomically, which would put on the appearance of general paresis ; but rather certain forms of the disease which have been variously named, but which is best known perhaps by its appellation of progressive locomotor ataxy.

I am fully disposed to believe that many cases of true locomotor ataxy have been considered to be general paresis. The diagnosis is easy enough when there are no cerebral symptoms; but, if any degree of mental disturbance existed in a case of ataxy, a mistake might be readily made. Some years ago, every case showing a degree of imbecility with impaired power of locomotion, would have satisfied the diagnosis of general paresis, and such cases would have been sent into the asylum. The removal of many of these cases into asylums has taken them from the observation of the general or hospital physician, and thus their frequency is not known to him ; while the asylum physician, seeing chiefly another phase of the disease, is less likely to recognise the true relationship of his cases with those described without mental disturbance. I have of late met with several cases of progressive locomotor ataxy which, at the first glance, might be easily taken for cases of paresis ; and some of them, indeed, have been so diagnosed by very competent men. I feel it to be the more important, therefore, that I should point out the distinction between these two diseases; and the diagnosis is of some consequence, for the future progress and duration of them differs considerably. The expectation of life in general paresis is from two to five years, while some cases of ataxy extend over ten, fifteen, and twenty years.

To avcid repetition, I will, in what is to follow, use the word ataxy instead of progressive locomotor ataxy, and paresis instead of general paresis (or paralysis) of the insane.

As I have already said, in typical cases without any mental disturbance, ataxy would not resemble paresis at all. The peculiar form of delirium, on the other hand-the délire des grandeur's-would, by its presence, pretty nearly decide that the case was one of paresis; but the fact is that, in certain cases of paresis, and in a certain stage of that disease, this symptom is not well marked.

There is also much variation in the symptoms of the disease, progressive locomotor ataxy. I will therefore give you a sketch of the forms in which it is to be met with, following M. Topinard in his work on the subject. I must premise, however, that what he describes is the disease as found in general hospitals. Nevertheless, he says that cerebral disturbance existed in several cases, and impairment of memory in one-tenth of the whole; and this I believe to be below the mark of cases generally. Earlier writers insisted much on the presence of certain symptoms as essential to the diagnosis of ataxy, such as the inability of the patient to walk blindfold; and some considered that the case was not ataxy without this symptom. More recent writers give the disease a much wider range; and, indeed, they will tell you that that peculiar symptom is by no means a frequent one.

M. Topinard, in his work L'Ataxie Locomotrice, makes a summary of the phenomena observed in 150 collected cases. I will quote his description at length, by way of presenting you a contrast to the account given in a former lecture of general paresis. He arranges the whole of his cases into ten groups; and those who have seen much of ataxy, will readily recognise most of these typical forms. In our clinical meetings, I shall be able to show you several of them.

M. Topinard's arrangement is as follows.

First Type. - The patient is ataxic in the lower or upper extremities. He staggers, and is more awkward when he closes his eyes, and there is little more than this to be observed.

Second Type.-Besides the above phenomena, the patient is not conscious of the movements he executes, or of the position of his extremities, and has some cutaneous anæsthesia.

Third Type.-The patient is ataxic more or less manifestly. In most cases there are pain in the back, a little numbness, some retention of urine, and more or less true paralysis, etc. But the symptoms are rigorously confined to the lower extremities.

Fourth Type.-The patient is plainly ataxic ; besides which, he has in his lower extremities excruciating nerve-pain (likened to the pain of toothache), of which the commencement may be traced sometimes to several years back; then come numbness, dulness of sensibility limited to the lower limbs, loss of sexual appetite, and pelvic complications (as want of control over the rectum and bladder). In general, there is no lumbar pain nor paralysis beyond that which one might describe as muscular weakness.

Fifth Type. - The nerve-pain (pain like toothache) continues some years, constituting, as in the previous type, a first stage of the affection; then there are added ophthalmic phenomena (amaurosis, diplopia, etc.); then follow embarrassment in speech, and even slight deafness or hardness of hearing, either transient or persistent. Next come anaphrodisia, and at length pelvic complications, which make up a second period. The numbness, ataxy, cutaneous and muscular anæsthesia (both more or less marked) attack the lower extremities, and later in the progress of the case the upper extremities. The commencement of the disease is insidious, its course irregularly progressive, and its duration indefinite.

Sixth Type.-The phenomena are the same as above (type 5), with the exception that the symptoms, or at least the principal symptoms, which characterise the first period, disappear in the second stage; thus suppressing or considerably diminishing the severity of the early symptoms.

Seventh Type.-The course and progress are the same as in the above, except that the commencement of the case showed itself by cerebral or spinal symptoms of acute or subacute kind ; the evolution of the disease continuing in other respects as in the two previous types.

Eighth Type.- The same phenomena as the above, except that the course of the disease is interrupted by cerebral and spinal complication.

Nintl $T y$ pe.-The same course as in $5,6,7,8$, only a complete or incomplete paralysis is added.

Tenth 7ype.-Obeying the course marked out in type 5, the case runs a more rapid course, and terminates by an abiolute paralysis. (DJ l'Ataxie Locomotrice, par Dr. Topinard, Paris, 1864 ).

Now, bearing in mind the phenomena as they present themselves to us in general paresis, and comparing them with the symptoms as given in M. Topinard's summary of locomotor ataxy, it would seem (I cannot in the present state of our knowledge use a stronger term) that the one set of phenomena began at one pole of the nervecentres, and the other at the opposite; the phenomena of the one belong chiefly to the cerebral, and the other chiefly to the spinal, system. While the phenomena are thus polarised, they appear distinct, and the differences can be readily observed; but as the disease extends, the one, as it were, from below upwards, and the other from above downwards, the syniptoms tend at the same time to assimilate or become of mixed character. There is, I think, in most cases of paresis at some stage a certain amount of ataxy or want of order ; while, on the other hand, in cases of ataxy there is some degree of paresis or want of power, and in not a few some mental imbecility, amnesia, stammering, and imperfect articulation, which give a case a kind of resemblance to one of paresis.

The history of the cases, however, will enable you to distinguish between these diseases, even at their most resembling period. In paresis, the mental symptoms are always present, and always precede the motor phenomena (if the motor symptoms ever precede the mental, as they 
are stated by some to do occasionally ; it is certainly very exceptional). The first symptoms in paresis are chiefly cerebral ; viz., mental excitement, great garrulity, noisy hilarity, bragging, early violence of behaviour, and very usually some exhibition of libidinous conduct ; and on the subsidence of excitement, the mind is found to be weak, and the motor phenomena gradually make their appearance.

On the other hand, in ataxy, to describe the case most likely to be confounded with paresis, the commencement is in the spinal functions. There is first an attack of pain of some remote part-of pain likened to the pain of toothache-occurring most frequently in the lower extremities, and dating several years back, considered at the time perhaps to be rheumatic ; this pain is worse towards evening, or when the patient is not mentally occupied; it may improve or disappear for a time and return. Then follows a slight degree of numbness of the part the patient feels as if he trod on wool ; occasionally " pins and needles" attack the part ; in fact, those phenomena which we have all experienced after sitting in an awkward position, when one's own leg has "gone to sleep." There is, as most of us know, want of feeling, want of recognition of the member, especially as to its size, and even it ownership, then atrocious pain and pins and needles. In the disease, on the subsidence of the pain, the patients exhibit some awkwardness in gait ; the ataxy or want of order on the movement is evident. These symptoms may extend over ten or twelve years with very little change. Except, perhaps, increasing awkwardness in gait, there is doubtless some numbness of the cutaneous surface in the course of the disease the phenomena appear to spread upwards by involving the functions of the nerves higher up ; the erection of the penis, and soon afterwards the sexual appetite are lost, and the disease ascends; the expulsory power of the bladder and rectum become impaired. All this occurs while little change takes place in the mental functions; but in other cases the mind appears imbecile, the memory is affected, and there is distinct alteration in behaviour and conduct; but there are no lofty ideas, no excessive excitement and garrulity, and in no case have I met with paroxysms of violence or libidinous ideas.

The differences may be better seen in a tabulated form.

$$
\text { Paresis }
$$

Runs its course in a few years.

Commences with mental symp toms.

Is attended with libidinuus ideas.

The motor symptoms are secondary in the order of time.

Is only rarely complicated with pelvic difficulties.

There often is great violence.
The treatment adopted, in all the cases except the last, was just that which I adopt in fracture of the clavicle. After pressing back the projecting end of bone as completely as possible, I apply a long strip of emplasirum roborans, to which pulvis opii or belladonna has been added, spread on leather, and drawn tightly obliquely from below upwards over the injury, and on over the top of the shoulder. This firm and tightly adhering plaster undoubtedly helps to keep the previously displaced parts in apposition, and also conceals from the notice of the patient any deformity at its worst period, when heightened by any swelling which may exist. I then place a wedge-shaped pad in the axilla, and apply the figure-of- 8 bandage; and finally take means to confine the elbow firmly to the side, and also to support it, and thereby to keep the shoulder from dropping.

My case of dislocation at the outer or acromial end occurred in December, 1871 , in a powerful young mason, who, while tipsy, was hustled out over the doorstep of one of our inns on to the pavement. In this case the end of the clavicle not only overlapped the acromion, but was so much tilted up, causing stretching and raising of the soft parts, that there was nearly an interval of an inch between it and the top of the acromion. In this case the treatment was the same as in the others, and the result was very satisfactory.

In reference to dislocations of the humerus at the shoulder, I have had a large number of cases, from various causes, or modes of violence. In one case a man was in the shaft of a mine, where the materials were being hauled up, standing on the ladder and further securing himself by holding by one hand to one of the bars, while he was performing some labour with his other hand, when a piece of wood fell down the shaft from the surface, descending upon the deltoid muscle of the limb by which he was holding, and thereby forcing the head of the humerus into the axilla. In this case the deltoid became so paralysed, and wasted away to such an extent, that the man did not recover the power to lift his arm, or the serviceable use of his limb, for quite fifteen months. I consider my mode of reducing these dislocations to be, in a great measure, new ; and I further consider it to be exceedingly neat and easy, as well as invariably successful in all recent cases. The best proof of this is, that I have never used chloroform or any other anæsthetic in any one of my cases. I place the patient (with the injured limb stripped of all clothing, of course) sideways on a strong chair, the sound side of the patient resting firmly against the back of the chair. I then place a jack-towel around the patient, as far up under the arm as convenient, and give the ends, either through the bars of the chair, or over the top of the back of it, into the hands of a strong attendant. I then fasten another towel around the injured limb just above the elbow, by the clove-hitch (or the simple reeve-knot, which does quite as well), and deliver the ends of this towel to another attendant, who, when in action, stoops or crouches somewhat. I instruct the first attendant, when I am ready, to fix one of his knees, or both, against the chair, and to keep the patient firmly fixed against the back of the chair, while the other assistant makes steady extension downwards and outwards from the elbow. Or I may put two attendants to the towel fastened above the elbow, if necessary. I then place myself by the side of the attendant (but towards the back of the patient) who is to make extension at the elbow. When all other matters are ready, I place both my thumbs on the point of the shoulder, at the end of the acromion, and press the fingers of each hand, the one before and the other behind the arm, into the axilla under and inside the head of the humerus. I cannot bring it down and out from where it is lodged by the force of my own hands, as I bring it against the neck of the scapula, or the rim of the glenoid cavity ; but almost immediately that I order the attendant, or attendants, having charge of the towel fastened above the elbow, to make steady extension downwards and outwards, I find the head of the bone to descend from its abnormal resting place, while $I$ assist and guide it, and simultaneously with its escape from within the rim of the glenoid fossa it springs into the cavity with a very audible snap. When this is done, and I find that the reduction is complete, by gentle movement of the limb forwards and backwards, with one of my hands on the point of the shoulder, and the other at the elbow, and by tilting up the arm, etc., I place a wedge-shaped pad in the axilla, fastening it in the usual way over and under the other shoulder, confine the elbow closely to the side, put the hand in a sling, order an embrocation to be anointed over the injured shoulder, and enjoin this rest of the limb for a week or more. I think I may correctly say that all my cases of dislocation of the shoulder have been downwards into the axilla.

My plan has never failed me in any recent case, and has never required, as before stated, either anzesthetics, bleeding, or other relaxing means. I cannot conceive anything less agreeable to bystanders, or the patient, than to place the patient on his or her back on the floor, and to stick your heel, especially if the stocking should not be quite sound or clean, into his or her armpit, and then to begin pulling and

* Read before the Surgical Section at the Annual Meeti ig of the British Medical Association in London, August 1873 . 